INFLAMMASOME

\title{
Starving inflammation
}

Inflammasomes have an important role in innate immunity, but their aberrant activation is linked to chronic inflammatory diseases. Reporting in Nature Medicine, Coll et al. and Youm et al. describe two compounds - MCC950 and $\beta$-hydroxybutyrate, respectively - that specifically inhibit the NLRP3 (NOD-, LRR- and pyrin domain-containing 3) inflammasome and have anti-inflammatory effects in mouse models of different NLRP3-dependent diseases.

Activation of the NLRP3 inflammasome leads to activation of caspase 1 and the release of the pro-inflammatory cytokines interleukin-1 $\beta$ (IL-1 $\beta$ ) and IL-18. NLRP3-mediated diseases are mainly treated with compounds that target IL-1, and Coll et al. investigated whether the IL- $1 \beta$ processing inhibitor MCC950 acts by inhibiting the inflammasome. Human and mouse macrophages were primed with lipopolysaccharide (LPS) and

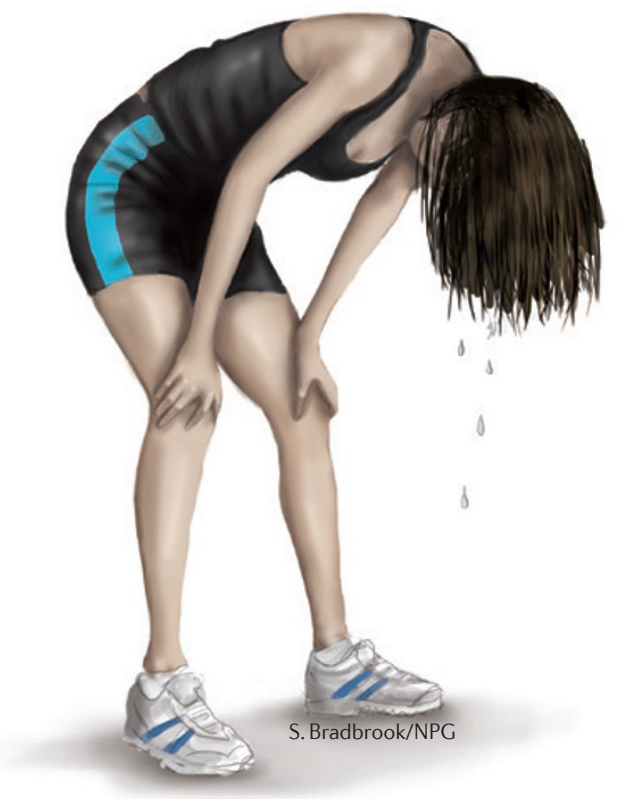

treated with MCC950 followed by stimulation with the NLRP3 activator ATP. In response to MCC950 treatment, the levels of both IL- $1 \beta$ and a caspase 1 autocatalytic cleavage fragment decreased in a dose-dependent manner. Moreover, the authors showed that MCC950 interferes with the formation of NLRP3-dependent ASC oligomers ('specks'), which is a key event in inflammasome activation. However, MCC950 did not prevent NLRP3 oligomerization or NLRP3-ASC interactions.

Youm et al. searched for endogenous mechanisms that could control NLRP3 deactivation. Ketone body $\beta$-hydroxybutyrate is produced in the liver and functions as an alternative energy source during fasting, lowcarbohydrate diets and high-intensity exercise, and as these states are associated with altered immune cell function, the authors investigated whether $\beta$-hydroxybutyrate could act as an immune effector. Indeed, $\beta$-hydroxybutyrate inhibited both ATP-induced cleavage of caspase 1 and the processing of the active form of IL- $1 \beta$ in mouse bone marrowderived macrophages (BMDMs). Additional experiments suggested that $\beta$-hydroxybutyrate specifically acts on a central signalling pathway that is specific to the NLRP3 inflammasome.

Prolonged fasting is associated with increased levels of $\beta$-hydroxybutyrate and reduced oxidative stress, which could regulate inflammasome activation. However, the inhibitory effects of $\beta$-hydroxybutyrate on NLRP3 were not dependent on starvationregulated mechanisms such as the production of reactive oxygen species and glycolytic inhibition. Instead, similar to the mechanism of action of MCC950, Youm et al. found that $\beta$-hydroxybutyrate inhibited ASC oligomerization and speck formation. Both research groups also investigated the effect of these inhibitors on $\mathrm{K}^{+}$efflux, which triggers NLRP3 inflammasome activation. Coll et al. found no effect of MCC950 on intracellular $\mathrm{K}^{+}$levels, whereas Youm et al. found that $\beta$-hydroxybutyrate prevented $\mathrm{K}^{+}$efflux in BMDMs in response to different NLRP3 activators.

Finally, both MCC950 and $\beta$-hydroxybutyrate were shown to have inhibitory effects in mouse models of the human Muckle-Wells syndrome, which is associated with a mutation in NLRP3.Interestingly, MCC950 was also shown to have an inhibitory effect in peripheral blood mononuclear cells from individuals with Muckle-Wells syndrome. Youm et al. found that feeding mice a ketogenic diet increased $\beta$-hydroxybutyrate levels in vivo, and that mice with a missense Nlrp3 mutation that leads to familial cold autoinflammatory syndrome - which is associated with neutrophilia - were protected from neutrophilia when fed a ketogenic diet compared with chow-fed mice.

In conclusion, both MCC950 and $\beta$-hydroxybutyrate specifically inhibit the NLRP3 inflammasome and could be promising treatments for a variety of NLRP3-mediated inflammatory disorders. Furthermore, the study by Youm et al. indicates that a low-carbohydrate ketogenic diet which leads to increased levels of $\beta$-hydroxybutyrate - could reduce the severity of NLRP3-mediated diseases. Elisabeth Kugelberg

ORIGINAL RESEARCH PAPERS Coll, R. C. et al. A small-molecule inhibitor of the NLRP3 inflammasome for the treatment of inflammatory diseases. Nature Med. http://dx.doi.org/10.1038/ nm.3806 (2015) | Youm, Y.-H. et al. The ketone metabolite $\beta$-hydroxybutyrate blocks NLRP3 inflammasome-mediated inflammatory disease. Nature Med. http://dx.doi.org/10.1038/nm.3804 (2015)

FURTHER READING Latz, E., Xiao, T. S. \& Stutz, A. Activation and regulation of the inflammasomes. Nature Rev. Immunol. 13, 397-411 (2013) 